\title{
Meaning in life assessed with the "Schedule for Meaning in Life Evaluation" (SMiLE): a comparison between a cancer patient and student sample
}

\author{
F. Stiefel • S. Krenz $\cdot$ C. Zdrojewski $\cdot$ D. Stagno • \\ M. Fernandez $\cdot$ J. Bauer $\cdot$ N. Fucina $\cdot$ F. Lüthi $\cdot$ \\ S. Leyvraz • G. D. Borasio • M. Fegg
}

Received: 31 July 2007 / Accepted: 12 December 2007 / Published online: 16 January 2008

(C) Springer-Verlag 2007

\begin{abstract}
Background The aims of the study were (a) to assess individual meaning in life (MiL) in a mixed sample of cancer patients with the Schedule for Meaning in Life Evaluation (SMiLE), (b) to evaluate the acceptability of its French version, and (c) to compare it to a student sample. Materials and methods Consecutive cancer patients $(N=$ 100) treated as outpatients in the University Hospital Lausanne $(N=80)$ and in a nearby hospice $(N=20)$ were evaluated with the SMiLE, a reliable and validated respondent-generated instrument for the assessment of MiL. The respondents list three to seven areas, which provide meaning to their life and rate the level of importance (weighting) and satisfaction of each area. Indices of total weighting (index of weighting (IoW), range
\end{abstract}

F. Stiefel $\cdot$ S. Krenz $\cdot$ C. Zdrojewski $\cdot$ D. Stagno

Psychiatry Service, University Hospital,

Lausanne, Switzerland

\section{Fernandez}

Fondation Rive-Neuve,

Villeneuve, Switzerland

J. Bauer $\cdot$ N. Fucina $\cdot$ F. Lüthi $\cdot$ S. Leyvraz

Pluridisciplinary Center of Oncology, University Hospital,

Lausanne, Switzerland

G. D. Borasio • M. Fegg

Interdisciplinary Center for Palliative Medicine,

University Hospital,

Munich, Germany

F. Stiefel $(\square)$

Service de Psychiatrie de Liaison,

Centre Universitaire Hospitalier Vaudois,

1011 Lausanne, Switzerland

e-mail: Frederic.stiefel@chuv.ch
20-100), total satisfaction (index of satisfaction (IoS), range $0-100$ ), and total weighted satisfaction (index of weighted satisfaction (IoWS), range 0-100) are calculated. Results Patients most often indicated areas related to relationships as providing MiL, while material things were listed less often. Since satisfaction with relevant areas was high, cancer patients reported the same level of weighted satisfaction (IoWS) as a healthy student sample, assessed with the SMiLE in a prior validation study. Patients judged the SMiLE as reflecting well their MiL, not distressing to fill in and were moderately positive with regard to its helpfulness.

Conclusions MiL of cancer patients was surprisingly high, possibly due to the "response shift" of the severely ill. The SMiLE might become a useful tool for research and an opener to communication between patients and clinicians about this highly relevant topic in cancer care. Further studies with larger sample sizes and different designs, complemented by qualitative research, are needed to deepen our understanding of this so characteristically human topic, which is so easy to perceive and so difficult to grasp.

Keywords Cancer - Meaning in life ·

Schedule for Meaning in Life Evaluation (SMiLE)

\section{Introduction}

The topic of "meaning in life" (MiL) has stimulated the interest of clinicians and researchers working in oncology. For example, Moadel et al. [1] who assessed cancer patients' most important needs found that $40 \%$ indicated a need for help in searching for MiL. A recent systematic literature review [2] of 44 studies on "meaning in the context of cancer" identified (a) studies focusing on the threatening and 
growth-enhancing aspects of cancer and the role of meaning attributed to the disease, (b) studies addressing the search for meaning, and (c) studies evaluating meaning as an outcome. The review concluded that there is strong empirical evidence of a relationship between meaning as an outcome measure and psychosocial adjustment to cancer. The authors deplored the lack of precise conceptual and operational definitions, the reliance on homogeneous sampling, and other methodological weaknesses in this field of research.

In clinical psychology, the concept of MiL has been introduced by Frankl [3], a psychiatrist who had a personal history as a survivor of the Nazi concentration camps. He defined MiL as the manifestation of values, which are based on (a) creativity (e.g., work, dedication to causes), (b) experience (e.g., nature, love), and (c) attitude (one's attitude toward suffering and existential problems). However, since Frankl's pioneering work, the interest in MiL has been very limited until it has been observed that loss of meaning is associated with psychological distress, the wish to hasten death and requests for euthanasia of cancer patients [4-7]. Consequently, psycho-oncological interventions focusing on MiL have therefore been developed [8-10].

The different questionnaires developed to assess MiL [11-28] measure intensity, but ignore the content of reported meanings [29], which vary from person to person and from situation to situation. Since measurements of MiL based on standardized models and pre-selected domains may not provide an adequate representation of this highly individual construct, the "Schedule for Meaning in Life Evaluation" (SMilE), based on a methodology utilized in quality of life (QoL) research [28], has been developed.

QoL assessment researcher faced similar problems, i.e., how to measure a highly individual concept, which is difficult to operationalize from a methodological point of view [29]. O'Boyle et al. therefore developed the "Schedule for the Evaluation of Individual Quality of life-Direct Weighting" (SEIQoL-DW) [30, 31]. In the SEIQoL-DW, the respondent indicates domains of individual QoL and rates their relative importance and satisfaction with each domain. The SMiLE was developed analogously to the SEIQoL methodology with the aim to provide an individualized assessment of MiL [28, 32].

This study aimed (a) to assess individual meaning in life (MiL) in a mixed sample of cancer patients by means of the SMiLE, (b) to evaluate the acceptability of its French version, and (c) to compare it to a student sample.

\section{Materials and methods}

The study has been approved by the Ethic Committee of the University Hospital Lausanne, Switzerland, and only patients who provided informed consent were included.
Patients

Consecutive eligible outpatients treated by the Pluridisciplinary Center of Oncology of the University Hospital Lausanne and inpatients of a nearby located palliative care hospice (RiveNeuve) were approached by their treating physician and asked for participation. Exclusion criteria consisted of insufficient knowledge of French and cognitive deficits hampering the assessment. Consenting patients were referred to the study nurse (CZ) for evaluation with the SMiLE.

\section{Schedule for Meaning in Life Evaluation (SMiLE)}

With the SMiLE, the respondents first indicate a minimum of three and maximum of seven areas ( $n=$ number of areas) which provide meaning to their life (step 1: area listing).

Then, the importance of each area $\left(w_{1} \ldots w_{n} ; 3 \leq n \leq 7\right)$ is rated with a five-point adjectival scale, ranging from 1 "somewhat important" to 5 "extremely important" (step 2: weighting).

Finally, the respondents rate their current level of satisfaction with each area $\left(s_{1} \ldots s_{n}\right.$; with $\left.3 \leq n \leq 7\right)$ on a seven-point Likert scale, ranging from -3 "very unsatisfied" to +3 "very satisfied" (step 3: level of satisfaction).

The index of weighting (IoW) indicates the mean weighting of the MiL areas (range 20-100, with higher scores reflecting higher weights). Since the scale starts with "somewhat important", the floor is set to 20 instead of 0 .

$\mathrm{IoW}=20 \circ \frac{w_{\text {ges }}}{n} ; w_{\text {ges }}=\sum_{i=1}^{n} w_{i}$.

The index of satisfaction (IoS) indicates the mean satisfaction or dissatisfaction with the MiL areas (range $0-100$, with higher scores reflecting higher satisfaction). To obtain an index varying from 0 to 100 , the satisfaction ratings $s_{i}$ are recalculated $\left(s_{i}^{\prime}\right)$. "Very unsatisfied" $\left(s_{i}=-3\right)$ is set to $s_{i}^{\prime}=0$ and "very satisfied" $\left(s_{i}=+3\right)$ is set to $s_{i}^{\prime}=100$ with levels of $16.7,33.3,50,66.7$, and 83.3 in between.

$\operatorname{IoS}=\frac{\sum_{i=1}^{n} s_{i}^{\prime}}{n}$.

In the total SMiLE index (index of weighted satisfaction, IoWS), the ratings for importance and satisfaction are combined (range 0-100, with higher scores reflecting higher MiL).

$\mathrm{IoWS}=\sum_{i=1}^{n}\left(\frac{w_{i}}{w_{\mathrm{ges}}} \circ s_{i}^{\prime}\right)$.

Levels and weights assigned to particular areas are independent. A person may be satisfied in a particular area but assign little importance to it, while another area may be 
described at a high level of both importance and satisfaction. Therefore an area perceived as "very unsatisfied" but of little importance will have less impact on MiL than an area that is "unsatisfied" but perceived as very important. This is reflected in the index of weighted satisfaction (total SMiLE index).

The psychometric properties of the SMiLE were evaluated in a study [28] with 599 students of the LudwigMaximilians-University, Munich, and the Royal College of Surgeons, Dublin (response rate 95.4\%). Mean IoW was $85.7 \pm 9.4$, mean IoS was $76.7 \pm 14.3$, and mean IoWS was $77.7 \pm 14.2$. Test-retest reliability of the IoWS was $r=0.72$ $(p<0.001)$, with $85.6 \%$ of all areas listed again after a testretest period of 7 days. Convergent validity was evaluated with the Purpose in Life test [22] $(r=0.48, p<0.001)$, the SelfTranscendence Scale [33] $(r=0.34, p<0.001)$, and a general NRS on MiL $(r=0.53, p<0.001)$. Convergent validity should not be too high, otherwise the need for a new instrument, except for reasons of acceptability and feasibility, is lacking. Discriminant validity has been tested with the Idler Index of Religiosity, showing no correlations between the two measures [28]. The psychometrics of the SMiLE were reported according to the recommendations of the Scientific Advisory Committee of the Medical Outcome Trust [34].

The translation procedure was conducted by independent forward and back translation of the SMiLE (German to French and French to German) by two bilingual German and French speakers.

Acceptability of the SMiLE was tested by Visual Analogue Scales (VAS), on which the patient was asked to indicate if the SMiLE "reflects what gives meaning to your live" (does not reflect $=0$ to reflects very well $=10)$, if it is "helpful" (not helpful $=0$ to extremely helpful $=10$ ), and if it is "distressing" to fill in (not at all distressing $=0$ to extremely distressing $=10$ ).

\section{Data analyses}

In statistical analysis, descriptive methods were used. Since a Kolmogorov-Smirnov test showed that the data were not normally distributed, we used nonparametric methods. To test for significant differences, the chi-square test and Mann-Whitney's $U$-test were used. Differences were considered to be statistically significant at $p=0.05$. Statistical tests were performed with the Statistical Package for Social Sciences (SPSS), version 15.0.

\section{Results}

Demographic and disease-specific characteristics

Of the 100 patients included, 80 were recruited in the University Hospital and 20 in the nearby palliative care hospice « Rive-Neuve ». Patients were evaluated for inclusion by the treating physicians, excluded or refusing patients were not documented. Mean age was 55years (SD $14.7)$, with about two thirds (69\%) being between 40 and 70years old; the majority were women (55\%), married (61\%), and Catholic or Protestant (91\%). Patients suffered from breast cancer $(30 \%)$, lung (18\%), uro-genital $(16 \%)$, gastro-intestinal (13\%), and other cancers (23\%), with $36 \%$ of them being considered as being « in remission ».

Item characteristics

The listed areas of the SMiLE were assigned to a posteriori categories, which were developed in the validation study [28].

Table 1 illustrates the frequency of MiL areas listed by the patients: "Family" was most often indicated, followed by "leisure time", "friends", and "partners", while "wellbeing", "altruism", "house/garden", and "finances" were least often indicated. Areas were weighted between 3.5 and 4.9 on the five-point Likert scale, with the highest levels of importance attributed to "family", "well-being", and "partners" and the lowest levels for "leisure time" and "work". With regard to satisfaction, areas were scored between 0.5 and 1.9 (scale ranging from -3 to +3 ), with "spirituality", "friends", and "family" scoring highest and "leisure time" and "finances" scoring lowest.

The table also summarizes a comparison of relevant results of the SMiLE between the patient and the student sample. Statistically significant differences were observed with regard to the frequencies of indicated areas. Patients were more likely to list "animals/nature", "leisure time", and "pleasure", while students were more likely to list "work", "friends", and "partner". No statistically significant differences were observed with regard to weighting and satisfaction with listed areas.

Index of weighting, satisfaction, and weighted satisfaction

Mean IoW was $85.9 \pm 7.2$, mean $\operatorname{IoS}$ was $77.3 \pm 15.5$, and mean IoWS was $78.1 \pm 15.1$ with no significant differences between the patient and student sample (IoW $85.7 \pm 9.4$, IoS $76.7 \pm 14.3$, IoWS $77.7 \pm 14.2$ ).

\section{Acceptability of the SMiLE}

Patients rated the SMiLE on a VAS (0-10) as "reflecting what provides meaning to your life" with a mean score of 7.0 (SD 2.8), and as "helpful" with a mean score of 4.5 (SD 3.2). With regard to the "distress" when filling in, a mean score of 3.6 (SD 1.9) was recorded, a lower score indicating that the SMiLE was perceived as "not at all distressing" to fill in. 
Table 1 Frequencies of listed MiL areas (percentage), weight and satisfaction in the sample of cancer patients and students

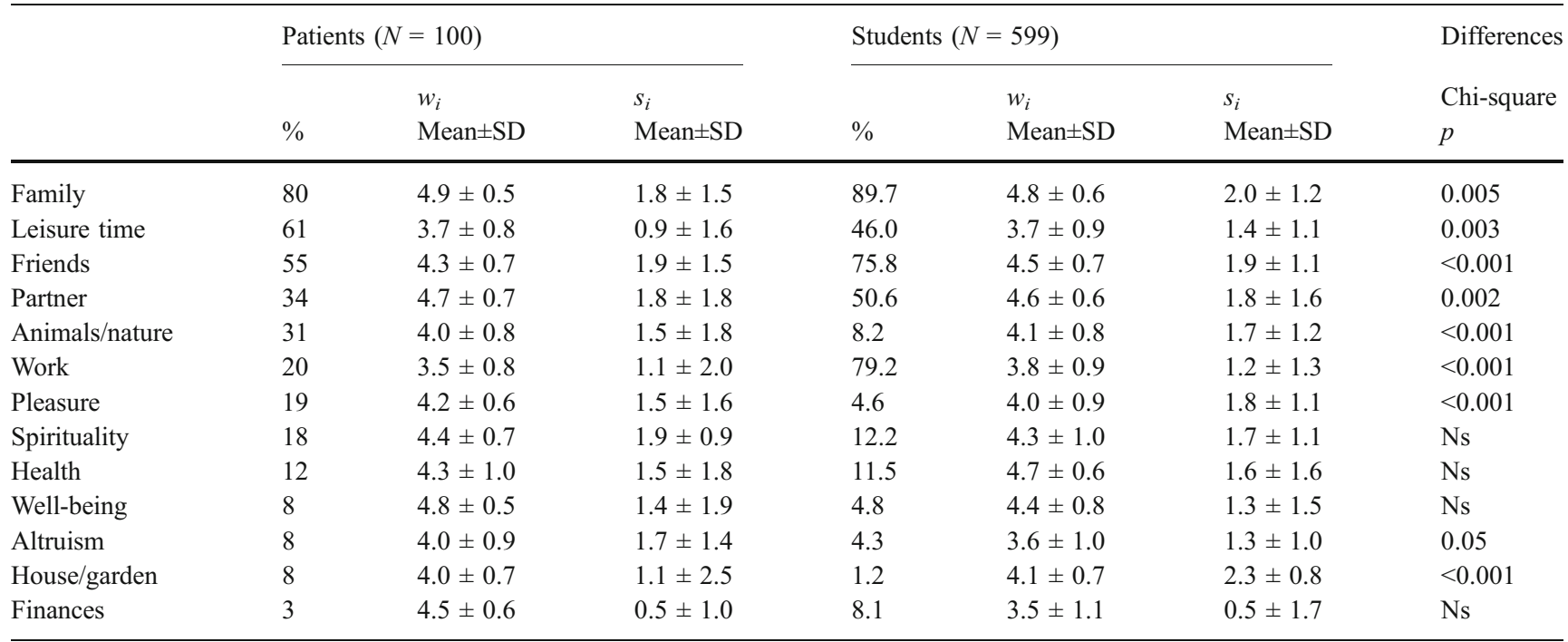

Significant differences between the samples with regard to frequencies of MiL areas (percentages) listed Ns Not significant

\section{Discussion}

This study aimed to assess MiL in a cancer patient population by means of the SMiLE. The areas mentioned by patients showed interesting discrepancies when compared to those mentioned by healthy students: "animals/ nature", "leisure time", and "pleasure" were rated higher by patients, while "work" was more often listed by the student sample. What is surprising is that "partner" is mentioned by a lower proportion in the cancer patient sample; this result may be due to the relatively high proportion of patients (about 40\%) reporting to be single, widowed, divorced, or separated, thus possibly indicating loneliness in a substantial proportion of patients. Satisfaction with areas identified by patients as important was high, which explains why cancer patients scored the same index of weighted satisfaction (IoWS) as healthy students. This can be understood as an expression of the "response shift" [35], a well-known phenomenon in the coping process of cancer patients who have to change their expectations towards life when confronted with the limitations of their disease. Beside the differences observed between the cancer patient and student sample, there are also many communalities leaving open, whether the measure and construct behind is an appropriate means to specifically capture individual MiL.

Given the objectives of the study, the sample size, and the many variables influencing MiL, statistical analyses were restricted to a descriptive analysis. Cancer patients indicated and attributed more weight to areas of MiL related to relationships (except for "partner", see comment above), while areas related to material things were listed less often. This confirms the clinical observation that for most patients confronted with a life-threatening disease, the feared or anticipated separation from material things is easier to accept than from relationships, which are often increasingly valued $[36,37]$.

With regard to acceptability, the SMiLE was perceived as reflecting rather well the concept of MiL, as not distressing to fill in, but as only moderately helpful. This may be due to the fact that, although the SMiLE allows an individual expression of MiL, it still represents a structured and quantitative assessment. Patients and investigators alike may share a feeling of ambivalence towards the attempt to "measure meaning in life". On one side, this seems to be a simplistic and "un-respectful" endeavor; on the other side, one has to remember that the same arguments have been put forward against quality of life research. If one understands that the SMiLE does not objectively "measures" MiL, but represents one possible way of investigating partial aspects of $\mathrm{MiL}$ and to introduce a clinically relevant concept into medical care, the ambivalence might diminish. As VAS measuring symptoms, such as pain or quality of life, the SMiLE may be utilized as a "door opener", which facilitates communication about existential issues for which patients are known to demand to be helped with [2]. We are also confirmed in our approach by quality of life research, which demonstrated that patient-centered, individualized, and weighted measures are adequate for assessing and evaluating highly individualized constructs [38]. From a scientific point of view, the SMiLE may be useful to deepen our understanding of MiL and to serve as an outcome measure, for example for psychotherapeutic interventions, which claim to be specifically designed to enhance MiL. 
A limitation of the study is the possible lack of representativeness, since recruitment was conducted by the treating physicians, who did not document excluded or refusing patients. However, types and stages of cancer, as well as sex and age distribution, correspond to a mixed cancer population. Since many variables, such as age, culture, stage of disease, or gender influence MiL, further studies with larger sample sizes and different designs, complemented by qualitative research, are needed to deepen our understanding of this so characteristically human topic, which is so easy to perceive and so difficult to grasp.

Acknowledgments This study was supported by a grant from OncoSuisse (No. 26840). The last author (MF) was supported by a scholarship of the Dr. Werner Jackstead Foundation (S134-10.007).

\section{References}

1. Moadel A, Morgan C, Fatone A, Grennan J, Carter J, Laruffa G, Skummy A, Dutcher J (1999) Seeking meaning and hope: selfreported spiritual and existential needs among an ethnicallydiverse cancer patient population. Psychooncology 8:378-385

2. Lee V, Cohen RS, Edgar L, Lainzner AM, Gagnon AJ (2004) Clarifying « meaning » in the context of cancer research: a systematic literature review. Palliative and Supportive Care 2:291-303

3. Frank1 V (1976) Man's search for meaning. Pocket, New York

4. Meier DE, Emmons CA, Wallenstein S, Quill T, Morrison RS, Cassel CK (1998) A national survey of physician-assisted suicide and euthanasia in the United States. N Engl J Med 338(17):1193-1201

5. Kissane D, Clark D, Street A (2001) Demoralization syndromerelevant psychiatric diagnosis for palliative care. J Palliat Care 17:12-21

6. Breitbart W, Rosenfeld B, Pessin H, Kaum M, Funesti Esch J, Galietta M, Nelson C, Brescia R (2000) Depression, hopelessness, and desire for hastened death in terminally ill cancer patients. JAMA 284:2907-2911

7. Meier D, Emmons C, Wallenstein S, Quill T, Morrison R, Cassel C (1998) A national survey of physician-assisted suicide and euthanasia in the United States. N Engl J Med 338:1193-1201

8. Breitbart W, Gibson C, Poppito SR, Berg A (2004) Psychotherapeutic interventions at the end of life: a focus on meaning and spirituality. Can J Psychiatry 49:366-372

9. Breitbart W, Heller K (2003) Reframing hope: meaning-centered care for patients near the end of life. J Palliat Med 6:979-988

10. Chochinov H, Hack T, Hassard T, Kristjanson L, McClement S, Harlos M (2005) Dignity therapy: a novel psychotherapeutic intervention for patients near the end of life. J Clin Oncol 23:5520-5525

11. Eckhardt P (2001) Skalen zur Erfassung von existentieller Motivation, Selbstwert und Sinnerleben. Existenzanalyse 1:35-39

12. Fife BL (1995) The measurement of meaning in illness. Soc Sci Med 40:1021-1028

13. Reker G (1992) Manual of the life attitude profile — revised. Student Psychologists, Peterborough (ON)

14. Salmon P, Manzi F, Valori RM (1996) Measuring the meaning of life for patients with incurable cancer: the Life Evaluation Questionnaire (LEQ). Eur J Cancer 32A(5):755-760

15. Hablas R, Hutzell RR (1982) The Life Purpose Questionnaire: An alternative to the Purpose-in-Life Test for geriatric, neuropsychiatric patients. In: Wawrytko SA (ed) Analecta Frankliana. Strawberry Hill, Berkeley (CA)

16. Battista J, Almond R (1973) The development of meaning in life. Psychiatry 36(4):409-427

17. Shapiro S (1976) Development of a life-meanings survey. Psychol Rep 39:467-480

18. DeVogler K, Ebersole P (1980) Categorization of college students' meaning of life. Psychol Rep 46:387-390

19. Ebersole P, Quiring G (1991) Meaning in life depth: the MILD. J Humanist Psychol 31:113-124

20. Warner S, Williams $J$ (1987) The meaning in life scale: determining the reliability and validity of a measure. J Chronic Dis 40(6):503-512

21. Starck P (1985) Guidelines - meaning in suffering test. Institute of Logotherapy, Berkeley (CA)

22. Crumbaugh J, Maholick L (1969) Manual of instructions for the Purpose-in-Life Test. Psychometric Affiliates, Munster (IN)

23. Reker G (2005) Meaning in life of young, middle-aged, and older adults: factorial validity, age, and gender invariance of the Personal Meaning Index (PMI). Pers Individ Differ 38:71-85

24. Wong P (1998) Implicit theories of meaningful life and the development of the personal meaning profile. In: Wong P, Fry P (eds) The human quest for meaning. A handbook of psychological research and clinical applications. Erlbaum, Mahwah (NJ), pp 111-140

25. Crumbaugh J (1977) The Seeking of Noetic Goals Test (SONG): a complementary scale to the Purpose in Life Test (PIL). J Clin Psychol 33:900-907

26. Antonovsky A (1993) The structure and properties of the sense of coherence scale. Soc Sci Med 36(6):725-733

27. Reker G (1996) Manual of the sources of meaning profilerevised (SOMP-R). Student Psychologists, Peterborough (ON)

28. Fegg MJ, Kramer M, L'hoste S, Hickey A, Borasio GD (2008) The Schedule for Meaning in Life Evaluation (SMiLE): validation of a new instrument in meaning-in-life research. J Pain Symptom Manage (in press)

29. O'Boyle C (1995) Making subjectivity scientific. Lancet 345:602

30. O'Boyle C, McGee H, Hickey A, O'Malley K, Joyce C (1992) Individual quality of life in patients undergoing hip replacement. Lancet 339:1088-1091

31. Joyce C, Hickey A, McGee H, O'Boyle C (2003) A theory-based method for the evaluation of individual quality of life: the SEIQoL. Qual Life Res 12:275-280

32. Fegg MJ, Kramer M, Bausewein C, Stiefel F, Borasio GD (2007) Meaning in life in the Federal Republic of Germany: results of a representative survey. Health Qual Life Outcomes 5:59

33. Reed P (1991) Self-transcendence and mental health in oldest-old adults. Nurs Res 40:5-11

34. Scientific Advisory Committee of the Medical Outcome Trust (2002) Assessing health status and quality-of-life instruments: attributes and review criteria. Qual Life Res 11:193-205

35. Schwartz C, Rapkin B (2004) Reconsidering the psychometrics of quality of life assessment in light of response shift and appraisal. Health Qual Life Outcomes 2:1-11

36. Stiefel F, Real O, Guex P (1998) An introduction to psychooncology with a special emphasis in its historical and cultural context. In: Portenoy RK, Bruera E (eds) Topics of palliative care. Oxford University Press, New York, pp 175-196

37. Guex P, Stiefel F, Rousselle I (2000) Psychotherapy with patients with cancer. Psychother Rev 2:269-273

38. Carr AJ, Higginson IJ (2001) Are quality of life measures patient centered? BMJ 322:1357-1360 